\begin{tabular}{ll} 
Behavior & Significance \\
\hline Number of & Yes, at red \\
Lionfish pectoral & snapper $(\mathrm{p}=0.019)$ \\
fin flares &
\end{tabular}

Percent time swimming tank
Yes $(p=0.002)$

\section{Gray Snapper}

Lane Snapper

Red Snapper

Lionfish

Lionfish flared at red

snapper significantly

more often than at

gray or lane snapper,

causing red snapper

to pause swimming.

Lionfish swam significantly more

than lane snapper in interaction trials;

significantly less

than lionfish in

interaction trials;

times in interaction

trials;

\section{No significant} difference among

gray snapper

swimming times in

gray-lionfish

interaction and

gray snapper

control trials.

\section{No significant}

difference among

lane snapper

swimming times in

lane-lionfish

interaction and

lane snapper

control trials.

\author{
When paired with When paired with \\ lionfish, red \\ snapper swam \\ significantly less \\ than in their 1- \\ individual and 4- \\ individual control \\ trials. \\ gray snapper, lionfish \\ swam significantly \\ less than in their 1- \\ individual control \\ trials; \\ No significant \\ difference among \\ lionfish swimming \\ times in lane-lionfish \\ or red-lionfish \\ interaction and \\ lionfish control trials.
}




\begin{tabular}{|c|c|c|c|c|c|}
\hline Behavior & Significance & Gray Snapper & Lane Snapper & Red Snapper & Lionfish \\
\hline \multirow[t]{2}{*}{$\begin{array}{l}\text { Percent time } \\
\text { swimming block }\end{array}$} & Yes $(\mathrm{p}=0.005)$ & $\begin{array}{l}\text { Although } \\
\text { minimal, gray } \\
\text { and lane snapper } \\
\text { swam } \\
\text { significantly more } \\
\text { at blocks than } \\
\text { lionfish in } \\
\text { interaction trials; }\end{array}$ & & $\begin{array}{l}\text { Although } \\
\text { minimal, red } \\
\text { snapper swam } \\
\text { significantly more } \\
\text { at blocks than } \\
\text { lionfish in } \\
\text { interaction trials; }\end{array}$ & $\begin{array}{l}\text { Lionfish swam } \\
\text { significantly less at } \\
\text { blocks than gray, lane, } \\
\text { and red snapper in } \\
\text { interaction trials; }\end{array}$ \\
\hline & & $\begin{array}{l}\text { No significant } \\
\text { difference among } \\
\text { gray or lane } \\
\text { snapper swimming } \\
\text { times at blocks in } \\
\text { gray-lionfish or } \\
\text { lane-lionfish } \\
\text { interaction and } \\
\text { gray or lane } \\
\text { snapper control } \\
\text { trials. }\end{array}$ & & $\begin{array}{l}\text { When paired with } \\
\text { lionfish, red } \\
\text { snapper swam } \\
\text { significantly more } \\
\text { at blocks than in } \\
\text { their 1-individual } \\
\text { control trials. }\end{array}$ & $\begin{array}{l}\text { No significant } \\
\text { difference among } \\
\text { lionfish swimming } \\
\text { times at blocks in } \\
\text { interaction and } \\
\text { control trials. }\end{array}$ \\
\hline $\begin{array}{l}\text { Percent time } \\
\text { huddling }\end{array}$ & Yes $(p=0.009)$ & $\begin{array}{l}\text { Although } \\
\text { minimal, } \\
\text { significantly } \\
\text { higher huddling } \\
\text { activity for gray } \\
\text { snapper in gray } \\
\text { snapper 4- } \\
\text { individual control } \\
\text { trials. }\end{array}$ & & $\begin{array}{l}\text { Although } \\
\text { minimal, } \\
\text { significantly } \\
\text { highest huddling } \\
\text { activity for red } \\
\text { snapper in lionfish } \\
\text { interaction trials. }\end{array}$ & \\
\hline
\end{tabular}




\begin{tabular}{|c|c|c|c|c|c|}
\hline Behavior & Significance & Gray Snapper & Lane Snapper & Red Snapper & Lionfish \\
\hline $\begin{array}{l}\text { Number of } \\
\text { approaches (or } \\
\text { retreats) }\end{array}$ & $\begin{array}{l}\text { Yes for lionfish } \\
(\mathrm{p}=0.034)\end{array}$ & $\begin{array}{l}\text { No significant } \\
\text { difference } \\
\text { between gray, } \\
\text { lane, and red } \\
\text { snapper } \\
\text { approaches and } \\
\text { retreats from } \\
\text { lionfish in } \\
\text { interaction trials. }\end{array}$ & & & $\begin{array}{l}\text { Lionfish approached } \\
\text { all three snapper } \\
\text { species more often } \\
\text { than they retreated } \\
\text { from them in } \\
\text { interaction trials. }\end{array}$ \\
\hline \multirow[t]{2}{*}{$\begin{array}{l}\text { Number of crabs } \\
\text { consumed per fish } \\
\text { Number of } \\
\text { predatory attempts } \\
\text { per fish }\end{array}$} & $\begin{array}{l}\text { Yes } \\
(p=0.007) \\
\text { Yes } \\
(p=0.016)\end{array}$ & $\begin{array}{l}\text { Gray snapper and } \\
\text { lane snapper } \\
\text { consumed and } \\
\text { attempted to prey } \\
\text { on significantly } \\
\text { less crabs than } \\
\text { lionfish in } \\
\text { interaction trials; }\end{array}$ & & $\begin{array}{l}\text { No significant } \\
\text { difference } \\
\text { between red } \\
\text { snapper and } \\
\text { lionfish prey } \\
\text { consumption or } \\
\text { predatory } \\
\text { attempts in } \\
\text { interaction trials; }\end{array}$ & $\begin{array}{l}\text { Lionfish consumed } \\
\text { and attempted to } \\
\text { prey upon } \\
\text { significantly more } \\
\text { crabs than gray and } \\
\text { lane snapper in } \\
\text { interaction trials; }\end{array}$ \\
\hline & & $\begin{array}{l}\text { No significant } \\
\text { difference among } \\
\text { gray or snapper } \\
\text { prey consumption } \\
\text { or predatory } \\
\text { attempts in gray- } \\
\text { lionfish } \\
\text { interaction and } \\
\text { gray snapper } \\
\text { control trials. }\end{array}$ & & $\begin{array}{l}\text { When paired with } \\
\text { lionfish, red } \\
\text { snapper } \\
\text { consumed and } \\
\text { attempted to prey } \\
\text { upon significantly } \\
\text { less crabs than in } \\
\text { their } 3 \text {-individual } \\
\text { control trials. }\end{array}$ & $\begin{array}{l}\text { No significant } \\
\text { difference between } \\
\text { lionfish and red } \\
\text { snapper prey } \\
\text { consumption or } \\
\text { predatory attempts in } \\
\text { interaction trials; }\end{array}$ \\
\hline
\end{tabular}




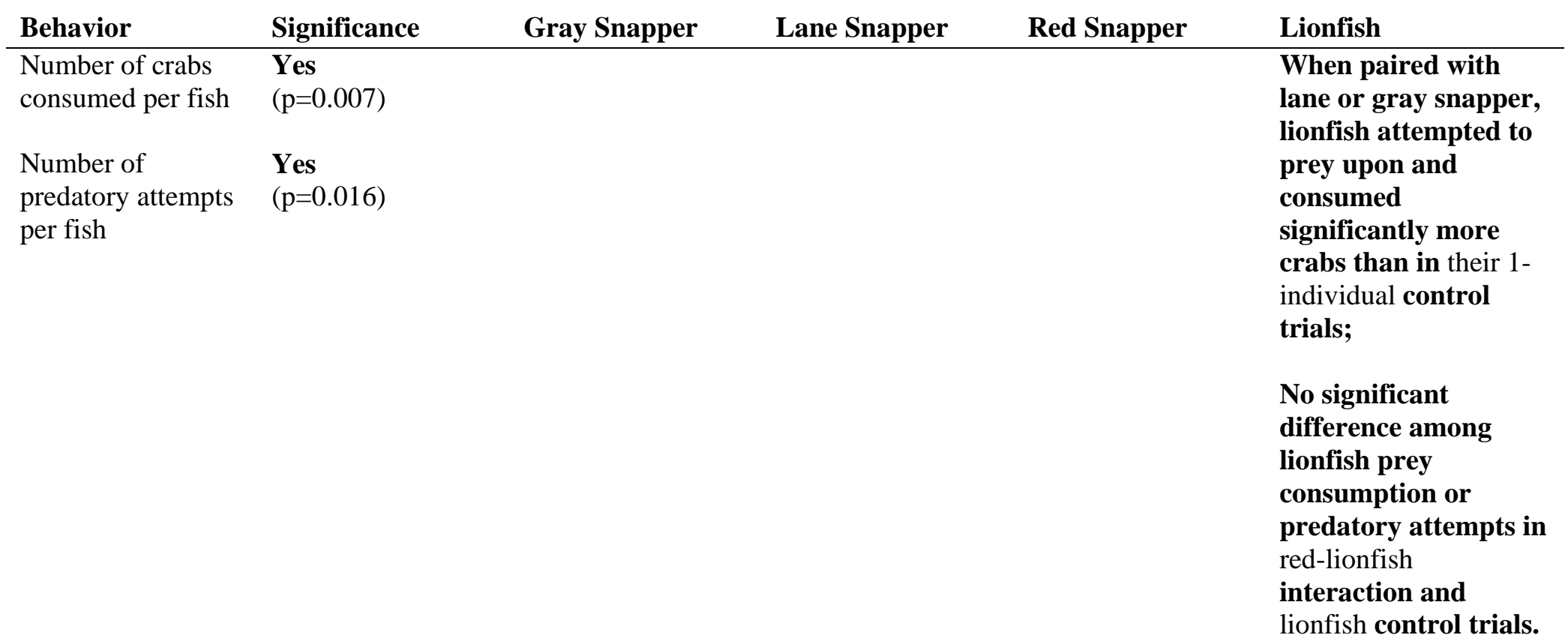

$\begin{array}{ll}\begin{array}{l}\text { Percent time spent } \\ \text { interacting }\end{array} & \begin{array}{l}\text { No } \\ (\mathrm{p}=0.629)\end{array} \\ \begin{array}{l}\text { Percent time } \\ \text { swimming at } \\ \text { center }\end{array} & \begin{array}{l}\text { No } \\ (\mathrm{p}=0.116)\end{array} \\ \begin{array}{l}\text { Percent time } \\ \text { pursuing prey }\end{array} & \begin{array}{l}\text { No } \\ \text { ( }=0.093)\end{array} \\ \begin{array}{l}\text { Number of prey } \\ \text { approaches (or } \\ \text { retreats) per fish }\end{array} & \begin{array}{l}\text { No } \\ (\mathrm{p}=0.086)\end{array}\end{array}$




\begin{tabular}{|c|c|c|c|c|c|}
\hline Behavior & Significance & Gray Snapper & Lane Snapper & Red Snapper & Lionfish \\
\hline $\begin{array}{l}\text { Number of times } \\
\text { prey was ignored } \\
\text { per fish }\end{array}$ & $\begin{array}{l}\text { No } \\
(p=0.607)\end{array}$ & & & & \\
\hline $\begin{array}{l}\text { Number of } \\
\text { aggressions per } \\
\text { fish }\end{array}$ & $\begin{array}{l}\text { No } \\
(p=0.628)\end{array}$ & & & & \\
\hline
\end{tabular}

